\section{Indicadores de salud mental asociados a riesgo psicosocial laboral en un hospital público}

\author{
ELISA ANSOLEAGA ${ }^{1,2}$
}

\section{Psychosocial stress among health care workers}

Background: Health care workers are exposed to high stress levels and psychosocial risks. The imbalance between the invested efforts and received rewards acquires special importance in this setting. Aim: To assess the psychosocial risk level and its relationship with depression, distress and psychotropic drug use among health care workers. Material and Methods: Seven hundred eighty two workers (602 females; 180 males) answered self-administered questionnaires to measure psychosocial risk and mental health. Results: Twenty five percent of respondents used psychotropic drugs, 34\% had a high level of distress and 23\% had depressive symptoms. They also reported a low level of decisional latitude (48\%), high emotional demands (47\%), low social support (41\%) and a signifcant effort-reward imbalance (67\%). Those exposed to job strain (high demands and low decisional latitude), iso-strain (job strain plus low social support), and effort-reward imbalance were twice as likely to suffer symptoms of depression and elevated distress compared to non-exposed subjects. Conclusions: There are high levels of psychosocial stress among health care workers.

(Rev Med Chile 2015; 143: 47-55)

Key words: Delivery of health care; Occupational health; stress, psychological.

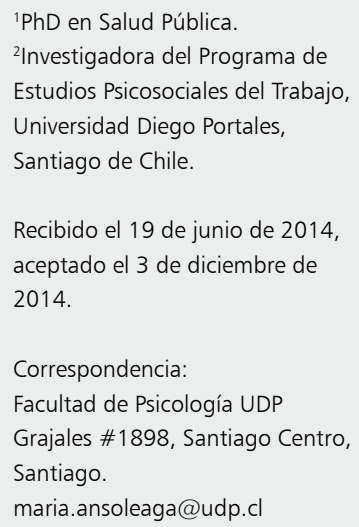

$\mathrm{E}$ 1 trabajo en el sector servicios con atención a personas implica particulares demandas y riesgos para la salud de los trabajadores ${ }^{1,2}$. La evidencia nacional e internacional ha descrito importantes niveles de estrés en trabajadores de la salud $^{3-6}$. Este trabajo es realizado principalmente por mujeres y, en consecuencia, atravesado por cuestiones de género, entre las cuales se observa una falta de diferenciación entre los espacios laborales de los no laborales ${ }^{7}$.

En este contexto laboral emergen los denominados riesgos psicosociales laborales, que aluden a un conjunto de características de la organización y de las condiciones de trabajo que afectan la salud de los trabajadores ${ }^{8}$. La exposición a estos riesgos tiene efectos importantes en la salud cardiovascular, osteomuscular y en la salud mental ${ }^{9-12}$. Dos son los modelos de riesgo psicosocial que acumulan mayor evidencia epidemiológica en el análisis de la salud mental laboral, el modelo Demanda/Control/Soporte de Karasek y Theorell ${ }^{13}$ y el modelo Desbalance Esfuerzos-Recompensas de Johannes Siegrist ${ }^{14}$. Ambos modelos analizan situaciones de la organización del trabajo que mediante mecanismos de estrés afectan la salud de los trabajadores. El modelo de Karasek y Theorell señala que trabajadores expuestos a altas demandas psicológicas combinadas con una escasa latitud decisional llevará a las personas a experimentar tensión psíquica, la que sostenida en el tiempo, puede repercutir en problemas de salud mental. Esta condición de riesgo se ve acrecentada cuando se le agrega un bajo soporte social, situación laboral descrita en la literatura por como isostrain. El modelo de Desbalance Esfuerzo-Recompensas trabaja bajo el supuesto de que las personas despliegan esfuerzos en sus trabajos y a cambio esperan recibir recompensas 
salariales, organizacionales y sociales. Cuando el esfuerzo invertido es mayor a las recompensas recibidas se produce una situación de desbalance, la que sostenida en el tiempo, generará emociones negativas y en el mediano plazo puede ser precursora de patología física o mental ${ }^{14,15}$. La exposición a estas situaciones de riesgo además de impactar en la salud de los trabajadores genera impactos organizacionales importantes relacionados con un mayor ausentismo; una mayor rotación del personal; menor satisfacción laboral, motivación y compromiso e incluso se ha vinculado a una mayor accidentabilidad y una peor calidad de la atención ${ }^{16-24}$. Atendiendo el importante impacto que los problemas de salud mental acarrean para las personas y las organizaciones, y que las dimensiones psicosociales laborales se han vinculado a la presencia de indicadores de salud mental, este estudio se propuso describir el nivel de riesgo psicosocial laboral y analizar sus asociaciones con sintomatología depresiva, distrés elevado y consumo de psicofármacos, en funcionarios de un hospital público de alta complejidad.

\section{Material y Método}

\section{Diseño, procedimientos e instrumento del estudio}

Con un diseño censal, este estudio de carácter analítico y transversal consideró la participación de 782 funcionarios (hombres $=180$ y mujeres $=$ 602) de un hospital pediátrico de alta complejidad, correspondiente al 70\% de la dotación efectiva.

Este estudio recibió aprobación del comité de ética de la Universidad Diego Portales. Los participantes firmaron un consentimiento informado resguardando garantías de voluntariedad y confidencialidad. El estudio fue difundido en las unidades del hospital con el fin de convocar a participar, explicitar los objetivos del estudio y los resguardos éticos. Estudiantes del Taller en Salud Mental Laboral de la Facultad de Psicología de la Universidad Diego Portales, colaboraron en la aplicación del instrumento, en parte de la difusión del estudio y de sus resultados. La dirección del hospital autorizó la realización del estudio pero no participó en su diseño.

Entre 2012 y 2013 se aplicó una encuesta autoadministrada (formato lápiz-papel), que incluyó dos escalas de riesgo psicosocial laboral: el cuestionario de Contenido de Trabajo (JCQ) que considera las variables demandas psicológicas, latitud decisional y soporte social, en su versión canadiense modificada para el contexto chileno con 16 ítems ${ }^{25}$; y la versión breve en español de la Escala de Desbalance Esfuerzo-Recompensa (ERI test 10 ítems) $)^{26}$, que considera esfuerzos, recompensas y desbalance. Ambos instrumentos fueron validados para Chile, en un estudio de validación con una muestra nacional, aleatoria y representativa de trabajadores chilenos ${ }^{27}$. Los instrumentos utilizan una escala de medida Likert con cuatro alternativas de respuesta ( 1 totalmente en desacuerdo y 4 totalmente de acuerdo). Asimismo, el instrumento consideró un screening de indicadores de salud mental y covariables de interés extraídas de otros estudios con poblaciones similares ${ }^{28,29}$. Las dimensiones psicosociales fueron dicotomizadas utilizando como punto de corte la mediana de la muestra en estudio.

\section{Variables independientes}

Demandas psicológicas: Conjunto de demandas cognitivas y psíquicas relacionadas con la cantidad, el volumen y/o la complejidad de las tareas, y de otras imposiciones (presión de tiempo, nivel de atención, interrupciones imprevistas), entre otras. Los puntajes oscilan entre 5 y 20 , y el punto de corte: mediana $=14$ puntos.

Latitud decisional: Grado de libertad que las personas tienen para decidir cómo hacer su trabajo (autonomía decisional) y la influencia que tienen en organización del trabajo. También incluye autonomía con relación al uso y desarrollo de las propias competencias. Los puntajes oscilan entre 5 y 20, y el punto de corte: mediana $=14$ puntos.

Soporte social: Apoyo instrumental (para realizar el trabajo) y emocional (para tramitar cuestiones no laborales en el trabajo) de colegas y superiores. Los puntajes oscilan entre 8 y 32 , y el punto de corte: mediana $=22$ puntos.

Esfuerzo extrínseco: Cantidad y ritmo de las tareas, y las interrupciones sufridas en el trabajo.

Recompensas: La recompensa social (estima y respeto), recompensas organizacionales (promoción en el trabajo y estabilidad/seguridad) y recompensas económicas (salario).

Para determinar el Desbalance EsfuerzoRecompensa (DER) se utilizó la siguiente fórmula:

$$
\text { DER }=\frac{\text { Puntaje directo esfuerzo }}{\text { Puntaje directo Recompensas x } 0,4285}, 1
$$


Donde 0,4285 es la división de $3 / 7$ correspondiente a los ítems para esfuerzo y recompensa de la escala breve de Siegrist ${ }^{26}$.

Sobrecompromiso: El modelo de Siegrist integra a su vez una tercera variable correspondiente a un componente de índole más personal que denomina "overcommitment" (traducida al español como sobrecompromiso). Esta propone que las personas caracterizadas por un patrón motivacional de excesivo compromiso con el trabajo y una alta necesidad de aprobación tienen mayor riesgo de tensión derivada del intercambio asimétrico y su susceptibilidad a la frustración de la recompensa es superior.

Las variables fueron dicotomizadas usando la mediana de los valores alcanzados en esta población y fueron generadas nuevas variables provenientes de la combinación de las dimensiones existentes: Jobstrain (combinación de altas demandas psicológicas y baja latitud decisional); Isostrain (combinación de Jobstrain más bajo apoyo social); y Desbalance esfuerzo-recompensas donde puntajes mayores a 1 indican desbalance esfuerzo-recompensa.

\section{Variables dependientes}

Sintomatología depresiva: Aun cuando existen diversos instrumentos validados para screening de depresión en estudios epidemiológicos, tales como CES-D ${ }^{30}$ y el PHQ-9 ${ }^{31}$, por razones de brevedad del instrumento se optó por dos preguntas sobre síntomas relevantes para el diagnóstico clínico de depresión mayor según el manual diagnóstico DSM-IV, relativas a la pérdida de interés por la mayoría de las actividades de la vida y sentimiento de tristeza, por un tiempo de dos semanas o más.

Distrés psicológico: Screening de distrés psicológico inespecífico que evalúa la prevalencia de síntomas en los últimos seis meses ${ }^{32}$. La escala K6 considera 6 síntomas asociados a depresión, inquietud, fatiga, sentimiento de culpa y ansiedad. Utiliza cinco alternativas de respuesta con puntajes donde 0 es nunca y 4 es siempre. Puntos de corte por categoría: $0=\sin$ distrés; 1 a $4=$ distrés bajo; 5 a $6=$ distrés moderado; 7 a $12=$ distrés elevado; 13 a $24=$ distrés muy elevado. La variable fue dicotomizada en: trabajadores/as sin distrés o con distrés moderado, y trabajadores/as con distrés elevado o muy elevado ${ }^{25}$. Esta escala ha sido utilizado en encuestas de salud poblacional en Canadá ${ }^{33}$ Estados Unidos y Australia ${ }^{34}$ por su brevedad y sus bondades psicométricas.

Consumo de psicotrópicos: Prevalencia año de consumo de psicotrópicos. Evaluada con la siguiente pregunta: "Durante los últimos 12 meses, ha tomado de manera regular, es decir, todos los días o algunas veces por semana, medicamentos para: a) ¿disminuir la ansiedad o el nerviosismo?; b) ¿ayudar a dormir? y c) ¿remontar el ánimo tales como antidepresivos?". En respuestas afirmativas se consultó respecto de los motivos: “ $i$ Cree que fue por problemas derivados del trabajo, de problemas en la familia, de la combinación del trabajo remunerado más el trabajo doméstico u otros?".

\section{Covariables}

Se indagó en variables laborales-demográficas tales como estamento, edad, y en variables de salud tales como: diagnóstico de enfermedad crónica, de depresión (prevalencia vida) o haber sufrido un suceso vital estresante en los últimos seis meses tales como accidente, víctima de algún hecho de violencia o la muerte de un familiar cercano. La encuesta consideró también la percepción sobre el estado de salud, ofreciendo alternativas de respuesta likert que van desde excelente a pésima ${ }^{35}$. No se incluyeron otras variables sociodemográficas que podrían haber resultados útiles para el análisis para disminuir la sobrecarga de la aplicación del instrumento en los participantes.

\section{Análisis estadístico}

Los datos fueron analizados por sexo, se consideró el porcentaje y distribución de las variables estudiadas, y mediante un modelo multivariado de regresión logística (OR 95\% IC), se estimó la chance de ocurrencia de los indicadores de salud mental (síntomas depresivos, distrés y consumo de psicotrópicos) en presencia de riesgo psicosocial, controlando por covariables que fueron significativas en el análisis bivariado: sexo, suceso vital estresante, diagnóstico vida de depresión y enfermedad crónica. Todos los análisis se realizaron utilizando el software Stata 12.0.

\section{Resultados}

La confiabilidad de los instrumentos utilizados fue previamente testeada en un estudio nacional de validación ${ }^{27}$. Para esta muestra el alpha de Cronbach de Karasek fue $\alpha=0,75$ y del modelo 
Salud mental y riesgo psicosocial en trabajadores de hospital - E. Ansoleaga

Tabla 1. Descripción de la muestra

\begin{tabular}{|c|c|c|c|}
\hline & $\begin{array}{l}\text { Mujeres } \\
\text { n (\%) }\end{array}$ & $\begin{array}{c}\text { Hombres } \\
\text { n (\%) }\end{array}$ & $\begin{array}{c}\text { Total } \\
\text { n } \quad(\%)\end{array}$ \\
\hline Total & $602(77)$ & $180(23)$ & $782(100)$ \\
\hline $\begin{array}{l}\text { Rangos de Edad } \\
\qquad \begin{array}{l}18-30 \\
31-40 \\
41-50 \\
51 \text { y más }\end{array}\end{array}$ & $\begin{array}{l}167(28) \\
154(26) \\
139(24) \\
129(22)\end{array}$ & $\begin{array}{l}49(29) \\
37(22) \\
37(22) \\
48(28)\end{array}$ & $\begin{array}{l}216(28) \\
191(25) \\
176(23) \\
177(23)\end{array}$ \\
\hline $\begin{array}{l}\text { Participación por Estamentos } \\
\text { Administrativos } \\
\text { Auxiliares } \\
\text { Técnicos Paramédicos } \\
\text { Enfermeras/os } \\
\text { Médicos } \\
\text { Otros Profesionales de Salud }\end{array}$ & $\begin{array}{r}60(10) \\
29(5) \\
272(46) \\
117(20) \\
48(8) \\
60(10)\end{array}$ & $\begin{array}{l}27(15) \\
23(13) \\
45(25) \\
1 \quad(0,6) \\
38(22) \\
40(23)\end{array}$ & $\begin{array}{r}87(11) \\
52(7) \\
317(42) \\
118(16) \\
86(11) \\
100(13)\end{array}$ \\
\hline $\begin{array}{l}\text { Tipo de Contrato } \\
\text { Titular (indefinido) } \\
\text { Contrata (plazo fijo anual) } \\
\text { Honorarios (por servicios) } \\
\text { Otro }\end{array}$ & $\begin{array}{r}240(41) \\
296(51) \\
13 \quad(2) \\
33 \quad(6)\end{array}$ & $\begin{array}{r}78(45) \\
80(46) \\
2 \quad(1) \\
13 \quad(8)\end{array}$ & $\begin{array}{r}318(42) \\
376(50) \\
15 \quad(2) \\
46 \quad(6)\end{array}$ \\
\hline
\end{tabular}

Fuente: datos de investigación 2013.

de Siegrist $\alpha=0,70$. Asimismo, la escala K6 mostró un $\alpha=0,92$.

Como se puede apreciar en la Tabla 1, la proporción de mujeres es significativamente mayor a los hombres y se constata una importante participación de técnicos paramédicos $(42 \%)$ en la muestra del estudio (Tabla 2).

En la Tabla 3 se reportan diversos indicadores sobre el estado de salud de la población encuestada: uno de cada cuatro funcionarios $(25 \%)$ reportó consumir al menos un psicotrópico con una diferencia significativa entre hombres y mujeres ( $28 \%$ versus $17 \%)$. Al consultar por las razones del consumo $40 \%$ señaló consumir psicotrópicos por problemas derivados de las obligaciones del trabajo remunerado más las del trabajo doméstico, alcanzando en las mujeres $44 \%$ y en los hombres $25 \%$, mientras que $15 \%$ señaló que el consumo se debe exclusivamente a razones de su trabajo remunerado. Asimismo, uno de cada tres reportó distrés elevado o muy elevado (34\%) y uno de cada cinco manifestó sintomatología depresiva (23\%) con diferencias significativas entre las mujeres y los hombres ( $25 \%$ y $17 \%$ respectivamente). Al consultar por los motivos de la sintomatología depresiva y del distrés elevado, no se observaron
Tabla 2. Dotación hospital y proporción de su participación en la muestra del estudio

\begin{tabular}{|lcc|}
\hline & $\begin{array}{c}\text { Dotación } \\
\text { hospital } \\
\text { (\%) }\end{array}$ & $\begin{array}{c}\text { Muestra } \\
\text { (\%) }\end{array}$ \\
\hline Auxiliares & 4 & 7 \\
Administrativos & 8 & 11 \\
Médicos & 21 & 11 \\
Enfermeras y otros profesionales & 25 & 29 \\
Técnicos paramédicos & 40 & 42 \\
\hline Directivos & 2 & 0 \\
\hline
\end{tabular}

Fuente: datos de investigación 2013.

diferencias estadísticamente significativas entre hombres y mujeres, donde uno de cada cinco funcionarios lo atribuye exclusivamente al trabajo remunerado, mientras que uno de cada dos lo atribuye a la combinación de las obligaciones provenientes del trabajo doméstico y del trabajo remunerado (Tabla 3 ).

También analizamos otras situaciones de salud encontrando que uno de cada dos funcionarios 
Salud mental y riesgo psicosocial en trabajadores de hospital - E. Ansoleaga

Tabla 3. Salud General e indicadores de Salud Mental en funcionarios de un hospital público en Chile

\begin{tabular}{|c|c|c|c|c|}
\hline & $\begin{array}{c}\text { Mujeres } \\
\text { n (\%) }\end{array}$ & $\begin{array}{c}\text { Hombres } \\
\text { n (\%) }\end{array}$ & $\underset{\left(\chi^{2}\right)}{p}$ & $\begin{array}{l}\text { Total } \\
\text { n }(\%)\end{array}$ \\
\hline \multicolumn{5}{|l|}{$\begin{array}{l}\text { Estado de salud mental } \\
\text { Consumo de psicotrópicos }\end{array}$} \\
\hline Hipnóticos & $102(17)$ & $14(8)$ & 0,003 & $116(15)$ \\
\hline Ansiolíticos & $99(17)$ & $21(12)$ & 0,140 & $120(16)$ \\
\hline Antidepresivos & $73(12)$ & $6 \quad(3)$ & $<0,001$ & $79(10)$ \\
\hline Al menos 1 psicotrópico & $163(28)$ & $30(17)$ & 0,006 & $193(25)$ \\
\hline \multicolumn{5}{|l|}{ Razones del consumo de psicotrópicos* } \\
\hline Trabajo remunerado & $51(13)$ & $19(19)$ & 0,202 & $70(15)$ \\
\hline Trabajo doméstico & $10(3)$ & $5(5)$ & 0,251 & 15 (3) \\
\hline Trabajo remunerado + doméstico & $166(44)$ & $26(25)$ & $<0,001$ & $192(40)$ \\
\hline Problemas familiares & $35(9)$ & 4 (4) & 0,077 & 39 (8) \\
\hline Otros & $117(31)$ & $49(47)$ & 0,590 & $166(34)$ \\
\hline \multicolumn{5}{|l|}{ Distrés } \\
\hline Distrés elevado & $204(35)$ & $51(30)$ & 0,230 & $255(34)$ \\
\hline Sin distrés o con moderado y bajo distrés & $383(65)$ & $120(70)$ & & $503(66)$ \\
\hline \multicolumn{5}{|l|}{ Sintomatología depresiva } \\
\hline Presencia de anhedonia + tristeza & $150(25)$ & $29(17)$ & 0,017 & $179(23)$ \\
\hline \multicolumn{5}{|c|}{ Motivos de la sintomatología depresiva y del distrés** } \\
\hline Trabajo remunerado & $24(19)$ & $9(32)$ & 0,127 & $33(21)$ \\
\hline Trabajo doméstico & $2(2)$ & $2(7)$ & 0,095 & $4(3)$ \\
\hline Trabajo remunerado + doméstico & $63(50)$ & $11(39)$ & 0,305 & $74(48)$ \\
\hline Problemas familiares & $24(19)$ & $4(14)$ & 0,555 & $28(18)$ \\
\hline Otros & $13(10)$ & $2(7)$ & 0,608 & $15(10)$ \\
\hline \multicolumn{5}{|l|}{ Estado de salud general } \\
\hline Excelente & $75(13)$ & $33(19)$ & 0,035 & $108(14)$ \\
\hline Muy buena & $184(31)$ & $63(36)$ & 0,196 & $247(33)$ \\
\hline Buena & $277(47)$ & $60(35)$ & 0,004 & $337(44)$ \\
\hline Pasable & $44 \quad(7)$ & $15(9)$ & 0,599 & 59 (8) \\
\hline Mala & $10(2)$ & $2(1)$ & 0,616 & $12(2)$ \\
\hline Salud excelente, buena o muy buena & $536(91)$ & $156(90)$ & 0,788 & $703(91)$ \\
\hline Salud mala o pasable & $54(9)$ & $17(10)$ & 0,788 & 71 (9) \\
\hline \multicolumn{5}{|l|}{ Otras situaciones de salud } \\
\hline Consumo de analgésicos & $335(56)$ & $63(36)$ & $<0,001$ & $398(52)$ \\
\hline Suceso vital estresante & $191(33)$ & $43(26)$ & 0,075 & $234(32)$ \\
\hline Enfermedad crónica & $176(30)$ & $40(23)$ & 0,092 & $216(28)$ \\
\hline Prevalencia vida diagnóstico depresión & $249(42)$ & $31(18)$ & $<0,001$ & $280(37)$ \\
\hline
\end{tabular}

*50 Datos faltantes. **Este ítem sólo se incluyó en una muestra de la población encuestada.

consume de manera regular (todos los días o casi todos los días) analgésicos con diferencias mayores y significativas en el consumo de mujeres (56\% y $36 \%$ respectivamente), casi $40 \%$ reportó haber recibido el diagnóstico médico de depresión a lo largo de su vida ( $42 \%$ mujeres y $18 \%$ hombres), cerca de un tercio ha recibido el diagnóstico para una enfermedad crónica y reportó un suceso vital estresante. Finalmente, llama la atención de que la evaluación global de su salud es muy positiva. Ante la pregunta espontánea por su estado de salud, 91\% de los encuestados señala que su salud es excelente, buena o muy buena y tan sólo $9 \%$ señala que su salud es pasable o mala (Tabla 3 ).

Con relación a la exposición a riesgo psicosocial laboral observamos que $48 \%$ reporta una baja capacidad de tomar decisiones y de desplegar sus destrezas en el trabajo (latitud decisional). El 47\% 
reporta altas demandas psicológicas del trabajo considerando una diferencia estadísticamente significativa entre hombres y mujeres $(40 \%$ y 49\% respectivamente). Asimismo, 41\% de los funcionarios encuestados reportó un bajo soporte social de pares y de superiores, y uno de cada cinco trabajadores presentó jobstrain, situación laboral de riesgo para la salud caracterizada por la combinación de altas demandas psicológicas y una escasa latitud decisional. Asimismo, 15\% de la muestra encuestada presentó Isostrain, situación laboral que combina jobstrain más bajo soporte social. Finalmente $67 \%$ de los participantes mostró desbalance entre los esfuerzos que invierte en el trabajo y las recompensas que recibe a cambio (Tabla 4).

Finalmente, mediante un análisis multivariado de regresión logística -y controlando por las posibles variables intervinientes- analizamos las asociaciones entre la exposición a las dimensiones psicosociales laborales y los resultados de sintomatología depresiva, distrés y consumo de psicotrópicos. Tal como se puede apreciar en la Tabla 5, los funcionarios expuestos a jobstrain tienen dos y media veces una mayor probabilidad de presentar sintomatología depresiva, 3,3 veces mayor chance de presentar distrés elevado o muy elevado y 1,5 veces una mayor chance de consumo

Tabla 4. Prevalencias de exposición a riesgo psicosocial laboral en hombres y mujeres trabajadores de un hospital público de alta complejidad

\begin{tabular}{|c|c|c|c|c|}
\hline & $\begin{array}{l}\text { Hombres } \\
\text { n }(\%)\end{array}$ & $\begin{array}{l}\text { Mujeres } \\
\text { n }(\%)\end{array}$ & $\mathbf{p}$ & $\begin{array}{l}\text { Total } \\
\text { n }(\%)\end{array}$ \\
\hline \multicolumn{5}{|l|}{ Modelo Demanda-Control-Soporte } \\
\hline Baja Latitud Decisional & $84(49)$ & $283(49)$ & 0,75 & $367(48)$ \\
\hline Alta Demanda Psicológica & $70(40)$ & $283(49)$ & $0,05^{*}$ & $353(47)$ \\
\hline Bajo Soporte Social & $75(44)$ & $234(41)$ & 0,47 & $309(41)$ \\
\hline $\begin{array}{l}\text { JOBSTRAIN } \\
\text { (Altas Demandas + Baja Latitud Decisional) }\end{array}$ & $36(21)$ & $131(23)$ & 0,71 & $167(22)$ \\
\hline $\begin{array}{l}\text { ISOSTRAIN } \\
\text { (Altas Demandas + Baja Latitud Decisional + Bajo Soporte Social) }\end{array}$ & $21(13)$ & $87(15)$ & 0,36 & $108(15)$ \\
\hline \multicolumn{5}{|l|}{ Modelo Desbalance Esfuerzos-Recompensas } \\
\hline Altos Esfuerzos & $86(49)$ & $303(52)$ & 0,62 & $389(51)$ \\
\hline Bajas Recompensas & $78(47)$ & $295(52)$ & 0,26 & $373(51)$ \\
\hline Sobrecompromiso & $67(39)$ & $242(42)$ & 0,62 & $309(41)$ \\
\hline Desbalance Esfuerzos-Recompensas & $114(66)$ & $403(68)$ & 0,56 & $517(67)$ \\
\hline
\end{tabular}

*Diferencia estadísticamente significativa.

Tabla 5. Asociaciones entre salud mental de trabajadores de un hospital público de alta complejidad y la exposición a riesgo psicosocial laboral (ajustado por suceso vital estresante, enfermedad crónica, prevalencia vida con diagnóstico médico de depresión y sexo). $\mathrm{N}=782$

\begin{tabular}{|c|c|c|c|c|c|c|c|c|c|}
\hline & \multicolumn{3}{|c|}{$\begin{array}{l}\text { Sintomatología } \\
\text { depresiva }\end{array}$} & \multicolumn{3}{|c|}{$\begin{array}{l}\text { Distrés } \\
\text { elevado }\end{array}$} & \multicolumn{3}{|c|}{$\begin{array}{l}\text { Consumo } \\
\text { psicotrópico }\end{array}$} \\
\hline & OR & IC $95 \%$ & $\mathbf{p}$ & OR & IC $95 \%$ & $\mathbf{p}$ & OR & IC $95 \%$ & $\mathbf{p}$ \\
\hline \multicolumn{10}{|c|}{$\begin{array}{l}\text { Modelo Demanda-Control-Soporte } \\
\text { JOBSTRAIN (Altas Demandas + Baja }\end{array}$} \\
\hline $\begin{array}{l}\text { Latitud Decisional) } \\
\text { ISOSTRAIN (Altas Demandas + Baia }\end{array}$ & 2,52 & $(1,66-3,80)$ & $<0,001$ & 3,38 & $(2,32-4,92)$ & $<0,001$ & 1,58 & $(1,03-2,43)$ & 0,026 \\
\hline Latitud Decisional + Bajo Soporte Social) & 2,98 & $(1,87-4,77)$ & $<0,001$ & 3,33 & $(2,14-5,17)$ & $<0,001$ & 1,59 & $(0,97-2,61)$ & 0,072 \\
\hline \multicolumn{10}{|c|}{ Modelo Desbalance Esfuerzos-Recompensas } \\
\hline Desbalance Esfuerzos-Recompensas & 2,16 & $(1,40-3,34)$ & $<0,001$ & 2,02 & $(1,40-2,89)$ & $<0,001$ & 1,27 & $(0,85-1,90)$ & 0,294 \\
\hline
\end{tabular}

Fuente: datos de investigación 2013. 
de psicotrópicos al compararse con los no expuestos. De otra parte, los expuestos a isostrain, tienen casi tres veces una mayor probabilidad de presentar síntomas depresivos $(\mathrm{OR}=2,98)$ y distrés muy elevado $(\mathrm{OR}=3,3)$, al compararlos con los sujetos no expuestos. Finalmente los expuestos a desbalance esfuerzos-recompensas tienen una chance dos veces mayor de presentar sintomatología depresiva $(\mathrm{OR}=2,16)$ y distrés muy elevado $(\mathrm{OR}=$ $2,02)$ al compararlos con los sujetos no expuestos a desbalance esfuerzos-recompensas (Tabla 5).

\section{Discusión}

Concordando con la evidencia internacional, el trabajo en servicios a personas en situación de sufrimiento, supone un conjunto de demandas relacionales y emocionales ${ }^{2,36}$ que pueden generar efectos nocivos en la salud de los trabajadores. A pesar de que uno de cada cuatro funcionarios encuestados consume psicotrópicos y uno de cada tres reporta distrés elevado, paradojalmente, $90 \%$ señala que su salud es muy buena, siendo posible hipotetizar, que en este grupo, la salud mental no es reconocida naturalmente como parte del estado de salud general.

Aún cuando las mujeres presentan peores indicadores de salud mental que los hombres, llama la atención que los hombres que trabajan en el hospital reportan peores resultados de salud mental que otras muestras de trabajadores o de hombres en población general. Utilizando los mismos métodos, una encuesta nacional reportó una prevalencia de síntomas depresivos de $5 \%$ en hombres; y aun cuando no sean metodológicamente comparables, el estudio de Araya encontró una prevalencia de síntomas depresivos de 2,7\% en hombres trabajadores del gran Santiago ${ }^{29,37}$, razón por la cual es relevante atender a los factores de la organización del trabajo que interfieren en su salud mental.

En este contexto de alta de exposición a riesgo psicosocial laboral, adquiere especial relevancia la dimensión de desbalance esfuerzos-recompensas. Además de los resultados adversos en la salud mental, el desbalance esfuerzos-recompensas es identificado como un factor contribuyente al riesgo de enfermedad coronaria ${ }^{17}$ y reconocido como un factor crítico en indicadores de índole organizacional en trabajadores de la salud: se ha vinculado a la decisión de renunciar o cambiar de profesión ${ }^{16}$, con episodios breves y frecuentes de ausentismo ${ }^{21}$, a una disminución de competencias laborales, el tiempo en la profesión, la violencia en el trabajo, entre otras ${ }^{22}$. Finalmente, las percepciones del ambiente de trabajo (apoyo social, carga de trabajo, sentimiento de pertenencia en el equipo y desbalance esfuerzo-recompensa) juegan un papel importante sobre los errores en la medicación y en la duración de la estadía de pacientes, impactando en la calidad del servicio/atención entregada ${ }^{23}$. A pesar de que estos elementos no fueron foco de este estudio, la magnitud de la prevalencia del desbalance esfuerzos-recompensas y de salud mental, pueden interferir con los requerimientos cognitivos, de atención y concentración que demanda el trabajo en salud y, en consecuencia, nos advierten de los efectos que la presencia de estos indicadores pueden tener en la calidad de la provisión del servicio.

Por otra parte, las mujeres tienen peores indicadores de salud mental y de riesgos psicosociales. Asumiendo la feminización del trabajo en salud dado que $70 \%$ son mujeres ${ }^{2}$, la consideración de la dimensión de género merece especial atención en futuras investigaciones.

Las fortalezas de este estudio radican en la cuantificación de los riesgos psicosociales y contar con un screening de salud mental en una muestra representativa de trabajadores de un hospital complejo, sobre la cual es posible realizar prevención y control. Entre las limitaciones, la tasa de rechazo de $30 \%$ acompañada de la falta de información sobre estos sujetos nos impide conocer si las características de quienes contestaron difieren de los que no contestaron. Sin embargo, con la sola excepción del estamento médico que está subrepresentado ( $11 \%$ vs $21 \%$ ), las proporciones de respuesta según estamento coinciden con su distribución en la dotación del hospital y la tasa de respuesta es superior a lo reportado en encuestas poblacionales sobre salud mental ${ }^{28,29}$. Otra limitación, por la necesidad de aplicar un instrumento breve, es la ausencia de otras dimensiones relevantes para analizar estos fenómenos tales como: las condiciones de trabajo, el sistema de turnos, la interferencia trabajo-familia, entre otras.

Hoy, resulta clave que los hospitales tomen conciencia que el trabajo en salud impacta en la salud de los trabajadores y como aquello puede además repercutir en indicadores organizacionales. El foco para enfrentar el estrés laboral debe transitar 
Salud mental y riesgo psicosocial en trabajadores de hospital - E. Ansoleaga

desde las vulnerabilidades individuales hacia los problemas de la organización del trabajo, donde la respuesta de intervención considere tres niveles (individuos, equipos y organización). A nivel organizacional, la instalación de mecanismos de prevención y control de los riesgos psicosociales, con medidas organizativas tales como: el diseño de puestos y sistemas de trabajo, un adecuado sistema de recompensas organizacionales y sociales (retribución); estándares para la protección de la salud psicológica en el trabajo: apropiados sistemas de comunicación con los usuarios, y acompañamiento a los funcionarios y equipos, para tramitar las demandas emocionales propias del trabajo en salud; la identificación y sensibilización en los grupos más expuestos a demandas emocionales, por ejemplo, los que trabajan en oncología o cardiovascular.

A nivel de los equipos, es de responsabilidad de las jefaturas mejorar sus sistemas de comunicación, retroalimentación y planificación del trabajo. En el nivel individual, la organización debe garantizar las condiciones para el reintegro al trabajo luego de una licencia prolongada por salud mental.

Agradecimientos: Al Hospital Luis Calvo Mackenna por su constante preocupación por la salud de sus trabajadores y por el apoyo brindado a este proyecto de investigación, en especial al Jefe de Desarrollo Organizacional, Jonathan Linares, por su constante colaboración. Al Proyecto Araucaria (Realizado por el Centro de Estudios de la Mujer en Chile y Financiado por el Global Health Research Initiative, Canadá) por el apoyo a esta iniciativa de Investigación. A la Facultad de Psicología y a los estudiantes del Taller de intervención colectiva en salud mental laboral de la Escuela de Psicología UDP por su apoyo en la recolección de datos y en la difusión del estudio y de los resultados. A la Federación Nacional de Trabajadores de la Salud (FENATS) con sede en el Hospital Luis Calvo Mackenna y a todos los trabajadores que estuvieron disponibles para colaborar en este estudio.

\section{Referencias}

1. Peiro JM, Peiró JM (2004). El sistema de trabajo y sus implicaciones para la prevención de riesgos psicosociales en el trabajo. Universitas Psychologica 2004; 3 (2): 17986.
2. Ansoleaga E, Artaza O, Suárez J, editors. Personas que cuidan personas: dimensión humana y trabajo en salud. OPS/OMS: Chile. ed: OPS/OMS: Chile; 2012.

3. Avendaño C, Bustos P, Espinoza P, García F, Pierart T. Burnout y apoyo social en personal del servicio de psiquiatría de un hospital público. Ciencia y enfermería 2009; 15 (2): 55-68.

4. Avendaño C, Román J. Efectos de los roles múltiples en el bienestar psicológico en enfermeras chilenas. Concepción: Universidad de Concepción, 2002.

5. Arenas F, Andrade V. Factores de riesgo psicosocial y compromiso (engagement) con el trabajo en una organización del sector salud de la ciudad de Cali, Colombia. Acta Colombiana de Psicología 2013; 16 (1): 43-56.

6. Acevedo G, Sánchez J, Farías MA, Fernández A. Riesgos Psicosociales en el Equipo de Salud de Hospitales Públicos de la Provincia de Córdoba, Argentina. Ciencia y Trabajo 2013; 15 (48): 140-7.

7. Díaz X, Mauro A. Riesgos psicosociales y salud mental de trabajadores y trabajadoras del sector salud en Chile. In: Ansoleaga E, Artaza O, Suárez J, editors. Personas que cuidan personas: dimensión humana y trabajo en salud. Santiago OPS/OMS: Chile; 2012. p. 146-64.

8. OIT-OMS. Factores psicosociales en el trabajo: naturaleza, incidencia y prevención. Ginebra: Organización Internacional del Trabajo. Organización Mundial de la Salud, 1984.

9. Vézina M. Stress au travail et santé psychique: différentes aproches. In: Neboit M, Vezina M, editors. Stress au travail et santé psychique. Toulouse: Octares; 2002.

10. Vézina M, Bourbonnais R, Brisson C, Trudel L. La mise en visibilité des problèmes de santé liés à l'organisation du travail. In: Brun J-P, P.-S F, editors. La santé et la sécurité du travail: problématiques en émergence et stratégies d'intervention Collection santé et sécurité du travail Quebec: PUL; 2008. p. 11-26.

11. Jolivet A, Caroly S, Ehlinger V, Kelly-Irving M, Delpierre $\mathrm{C}$, Balducci F, et al. Linking hospital workers' organizational work environment to depressive symptoms: A mediating effect of effort-reward imbalance? The ORSOSA study. Social Science and Medicine 2010; 71 (3): 534-40.

12. Gigantesco A, Lega I. Occupational stress and mental health. Epidemiologia e prevenzione 2013; 37 (1): 67-73.

13. Karasek R, Theorell T. Healthy work: stress, productivity, and the reconstruction of working life. New York: Basic Books; 1990.

14. Siegrist J. Adverse health effects of high efforts/low reward conditions. Journal of Occupational Health Psychology 1996; 1 (1): 27-41.

15. Siegrist J, Rodel A. Work stress and health risk behavior. 
Scand J Work Environ Health 2006; 32 (6): 473-81. Epub 2006/12/19.

16. Derycke H, Vlerick P, Burnay N, Decleire C, D'Hoore $\mathrm{W}$, Hasselhorn $\mathrm{H}-\mathrm{M}$, et al. Impact of the effort-reward imbalance model on intent to leave among Belgian health care workers: A prospective study. Journal of Occupational and Organizational Psychology 2010; 83 (4): 879-93.

17. Xu W, Hang J, Gao W, Zhao Y, Li W, Wang X, et al. Association between effort-reward imbalance and glycosylated hemoglobin (HbAlc) among Chinese workers: results from SHISO study. Int Arch Occup Environ Health 2012; 85 (2): 215-20.

18. Lamy S, De Gaudemaris R, Lepage B, Sobaszek A, Caroly S, Kelly-Irving M, et al. The Organizational Work Factors' Effect on Mental Health Among Hospital Workers Is Mediated by Perceived Effort-Reward Imbalance Result of a Longitudinal Study. J Occup Environ Med 2013; 55 (7): 809-16.

19. M. DCF, W. dC, V. dAC, L. N-M. Preliminary study about occupational stress of physicians and nurses in pediatric and neonatal intensive care units: the balance between effort and reward. Rev Lat Am Enfermagem 2010; 18 (1): 67-72.

20. Liu L, Chang Y, Fu J, Wang J, Wang L. The mediating role of psychological capital on the association between occupational stress and depressive symptoms among Chinese physicians: a cross-sectional study. BMC Public Health 2012; 12: 219.

21. Schreuder J, Roelen C, Koopmans P, Moen B, Groothoff J. Effort-reward imbalance is associated with the frequency of sickness absence among female hospital nurses: A cross-sectional study. International Journal of Nursing Studies 2010; 47 (5): 569-76.

22. Fischer F, Martínez M. Individual features, working conditions and work injuries are associated with work ability among nursing professionals. Work 2013; 45 (4): 509-17.

23. Paquet M, Courcy F, Lavoie-Tremblay M, Gagnon S, Maillet S. Psychosocial work environment and prediction of quality of care indicators in one Canadian health center. Worldviews Evid Based Nurs 2013; 10 (2): 82-94.

24. Bourbonnais R, Brisson C, Vézina M. Long-term effects of an intervention on psychosocial work factors among healthcare professionals in a hospital setting. Occupational and Environmental Medicine 2011; 68 (7): 479-86.

25. IRSST, INSPQ, ISQ. EQCOTESST Enquête Québécoise sur les Conditions de Travail, d'Emploi, de Santé et
Sécurité du Travail. Québec2010; Available from: http:// www.irsst.qc.ca/.

26. Siegrist J, Wege N, Puhlhofer F, Wahrendorf M. A short generic measure of work stress in the era of globalization: effort-reward imbalance. Int Arch Occup Environ Health 2009; 82 (8): 1005-13. Epub 2008/11/20.

27. Ansoleaga E, Montaño R, Vézina M. Validation of two complementary instruments for measuring work stress in Chilean workers. Scandinavian Journal of Organizational Psychology 2013; 5 (2): 5-14.

28. Chile Gd. Primera encuesta nacional de empleo, trabajo, salud y calidad de vida de los trabajadores y trabajadoras en Chile (ENETS 2009-2010). Santiago: Ministerio de Salud. Ministerio de Trabajo 2011.

29. CEM. U_Ottawa. Encuesta Araucaria. Santiago de Chile: Centro de Estudios de la Mujer 2011.

30. Gempp R, Thieme C. Efecto de diferentes métodos de puntuación sobre la fiabilidad, validez y puntos de corte de la escala de depresión del Centro para Estudios Epidemiológicos (CES-D). Ter Psicol 2010; 28 (1): 5-12.

31. Baader T, Molina J, Venezian S, Rojas C, Farías R, FierroFreixenet $\mathrm{C}$, et al. Validación y utilidad de la encuesta PHQ-9 (Patient Health Questionnaire) en el diagnóstico de depresión en pacientes usuarios de atención primaria en Chile. Rev Chil Neuro-Psiquiat 2012; 50 (1): 10-22.

32. Kessler RC, Andrews G, Colpe LJ, Hiripi E, Mroczek DK, Normand SL, et al. Short screening scales to monitor population prevalences and trends in non-specific psychological distress. Psychol Med 2002; 32 (6): 959-76. Epub 2002/09/07.

33. Statistique_Canada. Enquête sur la santé dans les collectivités canadiennes (ESCC): Questionnaire de 2007.

34. Furukawa T, Kessler R, Slade T, Andrews G. The performance of the K6 and K10 screening scales for psychological distress in the Australian National Survey of Mental Health and Well-Being. Psychological Medicine 2003; 33 (2): 357-62.

35. Bowling A. Just one question: If one question works, why ask several? J Epidemiol Community Health 2005; 59: 342-5.

36. Ansoleaga E, Toro J. Salud mental y naturaleza del trabajo: cuando las demandas emocionales resultan inevitables. Psicologia: Organizações e Trabalho 2014; (en prensa).

37. Araya R, Rojas G, Fritsch R, Acuna J, Lewis G. Common mental disorders in Santiago, Chile: prevalence and socio-demographic correlates. The British journal of psychiatry: the journal of mental science $2001 ; 178$ : 22833. Epub 2001/03/07. 